\title{
Using Browser Data to Understand Desires to Spend Time Online
}

Jesse D. McCrosky (joint first)

Mozilla Foundation

Douglas A. Parry (joint first) *

Stellenbosch University

Craig J. R. Sewall

University of Pittsburgh

Amy Orben

University of Cambridge 


\section{Author Notes}

\section{Affiliations:}

Jesse D. McCrosky: Mozilla Foundation; Douglas A. Parry: Department of Information Science, Stellenbosch University, South Africa; Craig J. R. Sewall: Department of Psychiatry, University of Pittsburgh, USA; Amy Orben: MRC Cognition and Brain Sciences Unit, University of Cambridge, UK

\section{Acknowledgments, funding sources, special circumstances:}

AO is supported by the UK Medical Research Council (SWAG/076.G101400) the UK Economic and Social Research Council (ES/T008709/1) and a Research Fellowship from Emmanuel College, University of Cambridge. CJRS is supported by the National Institute of Mental Health (T32MH018951).

We acknowledge the Mozilla Corporation for its support of the initial phases of this research and for the provision of the data for the study.

The data collection materials and analysis code are available on the OSF (https://osf.io/p3j7v/). Requests to access the synthetic data can be made via https://www.mrccbu.cam.ac.uk/bibliography/opendata/request/8782/.

The study was pre-registered prior to data analysis (https://osf.io/6wduk/).

\section{CRediT Statement:}

Conceptualization: Jesse McCrosky. Data curation: Jesse McCrosky. Formal analysis: Jesse McCrosky and Douglas Parry. Investigation: Jesse McCrosky. Methodology: Jesse McCrosky, Douglas Parry, Craig J. R. Sewall, and Amy Orben. Project administration: Jesse McCrosky, Douglas Parry, and Amy Orben. Writing - original draft: Jesse McCrosky, Douglas Parry, Craig J. R. Sewall, and Amy Orben. Writing - review \& editing: Jesse McCrosky, Douglas Parry, Craig J. R. Sewall, and Amy Orben.

\section{Corresponding author:}

Douglas A. Parry: dougaparry@sun.ac.za 


\section{Abstract}

There is growing recognition that many people feel the need to regulate their use of the internet and other digital technologies to support their wellbeing. In this study, we used Mozilla Firefox browser telemetry to investigate the role played by various usage factors in desires to regulate time spent online. In particular, we investigated how six metrics pertaining to time spent on the internet, and the diversity and intensity of use, predict participants' $(n=8,094)$ desires to spend more or less time online. Across all six metrics, we did not find evidence for a relationship between browser usage metrics and participants wanting to spend more or less time online. This finding was robust across various analytical pathways. The study highlights a number of considerations and concerns that need to be addressed in future industry-academia collaborations that draw on trace data or usage telemetry.

Keywords: Browser telemetry, Log data, Digital well-being, Mozilla Firefox, Trace data 


\section{Using Browser Data to Understand Desires to Spend Time Online}

Spurred by increasing media attention, the past decade has seen considerable debate among academics, policymakers, and the general public about how digital technologies impact our society and our well-being (Orben \& Przybylski, 2020; Parks, 2021; Twenge et al., 2020). Some have expressed concerns that high levels of digital technology use are causing addiction (Allcott et al., 2021; Rogers, 2021), decreases in cognitive function (Gazzaley, 2018), an oppressing attention economy (Harris, 2016), or decreases in well-being (Meier \& Reinecke, 2020; Twenge, 2018). From one perspective, such concern seems the natural response to new technologies changing our everyday behaviors (Orben, 2020b). Similar apprehension has followed when other technologies such as the radio or video games gained widespread popularity. Empirical evidence, however, does not support most concerns about negative effects associated with digital technologies (Johannes \& Dienlin, 2020; Meier \& Reinecke, 2020; Orben, 2020a; Valkenburg et al., 2022).

Recent qualitative research has drawn attention to individuals who have expressed desires to regulate their digital technology use (Grady et al., 2022; Marder et al., 2016; Orhan et al., 2021; Reinecke et al., 2021) and, for some, disconnect from modern digital technologies like the internet or social media (Chib et al., 2021; Jorge, 2019; Nguyen, 2021; Portwood-Stacer, 2013). Considering this, a growing body of research now focuses on the practices, motivations for, and effects of deliberate reduced or non-use of digital technologies (Hardey \& Atkinson, 2018; Kuntsman \& Miyake, 2019; Lomborg \& Ytre-Arne, 2021; Natale \& Treré, 2020). Such studies highlight various perceived negative effects associated with digital technology use (e.g., addiction, stress, anxiety). They also describe how these perceptions motivate attempts to 
regulate digital technology usage to improve users' well-being and avoid unwanted outcomes (e.g., privacy-violations, distraction, anxiety).

Disconnection from digital technologies is rarely total. As ever more aspects of life become digitized - education, entertainment, healthcare, commerce, communication-complete disconnection becomes increasingly difficult to achieve (Hesselberth, 2018; Kuntsman \& Miyake, 2019). Rather, like many other behaviors requiring self-regulation (Duckworth et al., 2019; Inzlicht et al., 2021), people are tasked with regulating their behavior to align their digital technology use with their general goals and desires, to ultimately minimize any negative effects that they may perceive to be associated with use. Recent work has drawn attention to the ambivalent and, at times, contradictory feelings people experience about their digital technology use-i.e., viewing time spent on digital technology as simultaneously rewarding and wasteful (Ytre-Arne et al., 2020). On the one hand, users are aware that digital technologies are sources of social interaction, entertainment, self-expression, and information, among many other uses and gratifications. On the other hand, users are also cognizant of the ways in which digital technologies can interfere with other goals or responsibilities (Nguyen, 2021; Parry et al., 2020). In support of achieving balance between the perceived harms and the perceived benefits of digital technology use, self-regulation may be achieved through various preventive or interventive strategies (Duckworth et al., 2016). Such steps, however, first require an individual to be aware of their own technology usage and the effects, if any, that this may have on other desired goals or experiences (Parry et al., 2020).

Against this backdrop, scholars have developed frameworks to understand the balance between the benefits and drawbacks of digital technologies and have focused on the dualities inherent in the complex array of uses and effects they enable (e.g., see Buchi, 2020; Vanden 
Abeele, 2020). Acknowledging that use of digital technologies can be both beneficial and detrimental, Vanden Abeele (2020) proposes the concept of "digital wellbeing" to focus on users' subjective evaluations of the optimal balance between the perceived negative and perceived positive effects associated with digital technology use. According to Vanden Abeele (2020, p.13) "people achieve digital wellbeing when experiencing maximal controlled pleasure and functional support, together with minimal loss of control and functional impairment." In line with this interpretation, individuals may seek to regulate or reduce their usage of a digital technology when they experience an imbalance between the perceived benefits and the perceived negative effects associated with its use.

To inform such theoretical approaches, in addition to focusing on the motivations and strategies that people use to regulate their digital technology usage (Chib et al., 2021; Nguyen, 2021; Parry et al., 2020), researchers have examined empirical evidence for positive or negative links between digital technology use and well-being (Dickson et al., 2018; Meier \& Reinecke, 2020). Progress in this regard has, however, been stymied by the absence of clear results and by debate over the meaning of study findings. While some argue that divergent results could be due to influential individual differences (Beyens et al., 2020; Valkenburg, Beyens, et al., 2021), others argue that extant research has suffered from substantial measurement issues (Ellis, 2019; Kaye et al., 2020; Parry et al., 2021; Sewall et al., 2020b). Specifically, most research on the uses and effects of digital technologies has relied on self-reported estimates of the time spent on, for instance, social media or the internet. However, due to several well-established cognitive and perceptual limitations (Schwarz \& Oyserman, 2001; Tourangeau, 1984), users have trouble providing accurate estimates of both their media use in general (Parry et al., 2021) and their internet use specifically (Araujo et al., 2017; Festic et al., 2021; Scharkow, 2016). 
In addition to concerns about the accuracy and ecological validity of self-report measures, researchers have also expressed concerns about the validity of the "screen time" construct — the aggregate total usage time on a platform, device, or application — that large proportions of the literature focus on (Kaye et al., 2020; Orben, 2020a; Parry et al., 2022; Valkenburg et al., 2022). Extant research tends to treat digital technology use as a uniform construct and simply focuses on the aggregate amount of time spent with digital technologies (Granic et al., 2020; Griffioen et al., 2020; Kaye et al., 2020). This approach neglects the many uses and gratifications that digital technologies enable, and ignores the important role played by various subjective, contextual, and content-specific aspects of usage (Parry et al., 2022). It is likely that such aggregate usage measures do not hold sufficient nuance to account for potential relations with well-being or desires to regulate time spent online.

The focus on screen time, coupled with a reliance on self-reported usage measures collected via surveys, has likely contributed to the slow development of suitable theories accounting for effects potentially associated with digital technologies, and desires to regulate time spent online (Orben, 2020b). These foci may have also precluded the consideration of alternative measures of digital technology usage. One potential way to kickstart a new phase of digital technology research is the collaboration and sharing of data and expertise between software companies and researchers. While challenging (e.g., Johannes, Vuorre, et al., 2021; Przybylski et al., 2021), such collaborations are key for improving measurement accuracy and diversifying the measures of digital technology use available to researchers (Orben et al., 2020).

Digital trace data (i.e., data that are produced and logged as a byproduct of digital technology use; Freelon, 2014) or telemetry (i.e., the collection of in-situ data for transmission to a receiver) collected by software companies can provide behavioral information about the time 
spent with digital technologies with a greater level of ecological validity compared to retrospective self-reports or simulation studies in laboratory environments. Such data may also enhance researchers' abilities to capture the diversity and intensity of use and move away from crude time-based measures. Diversity of use provides a proxy for the amount and variety of material accessed and may be approximated by, for instance, the number of unique domains an individual accesses. Similarly, the intensity of use-which indicates the amount of material accessed in a given time period — may be measured by the number of uniform resource identifiers (URIs) accessed per active hour in a specific period. Metrics like these that are computed on the basis of in-situ use of digital technologies may prove useful for understanding digital well-being and intentions to regulate digital technology usage as they provide insight into the nature of use and may relate to an individual's sense that their time has been "well-spent" and that their behavior aligns with some self-defined ideal or, in the case of usage diversity, a sense of cognitive overload and fatigue (Fu et al., 2020; Lin et al., 2020; Pelet et al., 2017).

\section{The Current Study}

Given the aforementioned measurement and conceptual issues, to advance our understanding of digital well-being in general and, in particular, to study the factors that may be associated with desires to self-regulate time spent online with an ecologically valid approach, the current study draws on data collected directly from real-world, in-situ browser usage sessions and focuses on a range of usage metrics beyond simply the duration of use. Specifically, we use such telemetry and survey data collected from a large sample in collaboration with the Mozilla Corporation to address the following research question: 
How does wanting to spend time online correlate with different quantifications of internet use devised from telemetry data?

\section{Method}

To address our research question, we used a mixed-method dataset collected from users of the Firefox browser. This dataset includes survey data about perceptions of time spent online, internet usage, and basic demographics, as well as linked in-situ telemetry that allows for the computation of various browser usage metrics.

To enable others to critically examine our work, while constrained by the proprietary nature of our dataset, we aimed to be as transparent as possible in our workflow. To guard against specification searching and distinguish between a priori and post-hoc analytical decisions, we specified a pre-analysis plan and registered it on the Open Science Framework (OSF) prior to data analysis (https://osf.io/6wduk/). At the time of registration, JDMC was familiar with the data so, for this reason, was not involved in the analytical choices made, and only provided guidance about the nature of the telemetry. AO had some exposure to the data (descriptive statistics) but had not had access to the data itself. DP and CS did not have access to any data, descriptive or inferential results, and were only provided with details of the study design. In addition to registering our analysis plan, while we are unable to share the original raw data for legal and ethical reasons, we have produced a synthetic dataset using the SynthPop package (Nowok et al., 2016; Reiter, 2005) that mimics the original dataset while protecting the privacy of participants. Synthetic data preserve the statistical properties of variables and the relationships between variables, but no record represents a real participant (Quintana, 2020). ${ }^{1}$ The data collection materials and analysis code are available on the OSF (https://osf.io/p3j7v/);

\footnotetext{
${ }^{1}$ See the supplementary methods for an explanation of the process used to produce the synthetic dataset.
} 
requests to access the synthetic data can be made via https://www.mrccbu.cam.ac.uk/bibliography/opendata/request/8782/.

\section{Participants and Procedure}

In September 2019 the Mozilla Corporation sent in-browser invitations to a randomly selected sample of users ${ }^{2}$ asking them to complete a survey hosted on SurveyGizmo. To be eligible to receive an invite, users had to use the Firefox web browser and had to have telemetry turned on. The survey included an identifier that, with the approval of the trust and legal departments at the Mozilla Corporation, enabled the survey responses to be linked to participants' browser telemetry. The invitation received 20,042 responses from 19,961 unique users and, after deduplication, removal of responses for which telemetry-linkage was impossible, or usage metrics could not be computed, resulted in an initial sample of $n=15,311$ participants with matched browser telemetry.

From this sample, following our pre-analysis plan, we excluded those who indicated that Firefox was not their primary browser $(n=2,680)$, or that their mobile internet usage is greater than their desktop usage $(n=3,724)$. This resulted in an eligible sample of $n=8,907$. Upon receiving the data, two further unplanned exclusion steps were required to produce the final sample. To calculate seven-day usage metrics we needed to remove participants who created their profiles less than seven days before data collection $(n=334)$, as well as those with implausible activity metrics ${ }^{3}(n=479)$. These procedures resulted in a final sample of $n=8,094$.

\footnotetext{
${ }^{2}$ To restrict to adult users, the invitation included a line that stated that "You must be 19 years of age or older to take part in this survey."

${ }^{3}$ This included participants with zero active days, zero active hours, zero URIs loaded, zero domains visited, or those whose telemetry indicated usage of more than 168 active hours in a week. These metrics are either physically impossible due to the number of hours in a week or, given that the browser needed to be used for the survey, cannot reflect actual browser usage, and likely represent measurement errors.
} 


\section{Measures}

Telemetry data. The Firefox Telemetry system (Mozilla, 2017) provides non-personal browser performance and usage data to Mozilla through a 'ping' sent approximately daily. Using these data the following metrics are recorded: (1) the number of active days in the last week (i.e., days that the Firefox installation was running and connected to the internet; Mozilla, 2021b); (2) the number of active hours in the last week (i.e., an aggregate of the number of five-second "ticks" in which the browser received keyboard or mouse activity; Mozilla, 2021a); (3) the number of URIs ${ }^{4}$ loaded in the last week (this metric can be interpreted as a proxy for the number of webpages loaded); and (4) the number of unique domains loaded in the last week (this metric can be interpreted as a proxy for the number of specific hosts visited e.g., Facebook.com, Google.com, Wikipedia.org, etc.). ${ }^{5}$ In addition to these four raw metrics, two additional derived variables were computed for each participant: (1) the number of URIs per active hour loaded in the last week and (2) the number of unique domains per active hour loaded in the last week.

Survey data. Three versions of the survey were designed (see the materials on the OSF for the full survey), with each sent to approximately one third of the participants. In the first version (completed by 2,702 participants in the final sample), to assess participants' feelings about their internet use, a single item was used to elicit their desire to spend more time online

\footnotetext{
${ }^{4}$ A Uniform Resource Identifier (URI) is a unique sequence of characters that identifies a resource. Some URIscalled Uniform Resource Locators (URLs) or web addresses - provide a means of locating and retrieving a resource on a network (e.g., the Internet). In a web browser, URLs are used to reference and retrieve a web page to be displayed in the browser. As such, the number of URIs loaded can be used as a proxy for the total number of webpages loaded.

${ }^{5}$ The Mozilla Corporation also recorded telemetry data for the last 28 days. For this study, as noted in our preregistration, we decided to only examine usage metrics recorded in the last week prior to survey completion rather than the last 28 days due to the state-like nature of the survey data which concern current feelings about wanting to spend more or less time online.
} 
("If I could, I would spend more time online"). In the second version of the survey (completed by 2,670 participants in the final sample), a single item was used to elicit participants' desires to spend less time online ("If I could, I would spend less time online"). In the third version of the survey (completed by 2,722 participants in the final sample) both versions of the item were presented. In all three versions responses were provided through a Likert scale ranging from Strongly Disagree (1) to Strongly Agree (5). Other than these differences, the remainder of the items were identical across the three survey versions.

Participants were asked to indicate from a list of options which browser was their primary browser on non-mobile devices. Two optional demographic items were used to elicit participants' age group and geographic region (i.e., continent). Finally, participants used a slider to indicate the proportion of their time online spent on a desktop device versus on a mobile device. In addition to these items, the survey assessed several measures not included in any of the pre-registered analyses reported in this study (see the supplementary methods for further information on these additional measures).

\section{Analytic Approach}

In addition to the application of the eligibility criteria and the removal of implausible usage data previously described, our analysis began by producing three calculated variables. The first twoURIs per active hour and unique domains per active hour - were calculated by dividing the respective variables by the total number of active browser usage hours in the observation period for each participant. Next, we used the responses for the items concerning desires to either spend more or less time online to calculate an overall variable that represented their "internet usage desire." Responses to the initial items were negatively correlated $\left(r_{s}=-0.55\right)$ and thus, following 
our pre-registered protocol, we considered them to be measuring the same construct but on opposite scales. ${ }^{6}$ Therefore, to produce an overall variable for internet usage desire, we created an integer-scaled variable for each ordinal measure and reverse-coded the "desire to spend less time online" item. For the "desire to spend more time online" question, we assigned 0 for "strongly disagree" and 4 for "strongly agree", etc. For the "desire to spend less time online" question, we assigned 4 for "strongly disagree" and 0 for "strongly agree", etc.

For those who received both items, we used the mean of these values, while responses for those who only received a single item were added as-is after reverse-coding. We considered the resulting value as an ordinal variable with 9 possible levels: $0,0.5,1, \ldots, 3.5$, 4 , with higher values indicating greater agreement with the desire to spend more time online. This variable formed the basis of our primary pre-registered analysis. However, as an additional sensitivity analysis, we also analyzed each original response direction (i.e., "desire to spend more time online" and "desire to spend less time online") separately.

We used ordinal logistic regression to examine the relationships between the internet usage metrics and internet usage desire. Specifically, we regressed internet usage desire on each of the six telemetry variables (i.e., the number of active days, the number of active hours, the URI count, the domain count, URIs per hour, and domains per hour) in separate models, while controlling for age category and region of residence. Due to very low rates of missing data for the demographic variables $(>97 \%$ of participants provided data on their region of residence and age), we did not perform any imputation for missing data as originally planned. To account for multiple testing, we used Bonferroni-adjusted confidence intervals. ${ }^{7}$

\footnotetext{
${ }^{6}$ In a sensitivity analysis we consider the possibility that these items represent two distinct constructs (see Figure 3 ).

${ }^{7}$ Bonferroni adjustments were made based on the six models specified in the pre-analysis plan, one for each of the primary independent variables of interest (days active, hours active, etc.). As such, the confidence intervals
} 
Following the primary analysis, we conducted several sensitivity analyses to determine the robustness of our results. Three of these were pre-registered and a fourth was conducted as a post hoc exploratory analysis. The first pre-registered sensitivity analysis accounted for the decision to remove participants who indicated that their mobile internet usage is greater than their desktop usage $(n=3,724)$. For this analysis the six ordinal logistic regression models were recalculated with the full sample $(n=11,476)$. The second pre-registered sensitivity analysis involved analyzing the three versions of the survey separately (i.e., separate models were produced for those who received either the "desire to spend less time online", item the "spend more time online", item or both items). Our third pre-registered sensitivity analysis involved analyzing the internet usage desire variables as two separate, directional variables. Notably, in our pre-analysis plan we specified that if the correlation between these two variables was greater than or equal to $a b s\left(r_{s}\right)=0.5$ we would combine them (using reverse scoring if necessary). However, while the threshold was passed $\left(r_{s}=-0.55\right)$, given the proximity of the correlation coefficient to the threshold, and the relatively low bound we specified, we ran a sensitivity analysis considering these as two distinct variables: "desire to spend less time online" and “desire to spend more time online." Our final sensitivity analysis was not pre-registered. Rather, given the skewness of the usage metrics produced from the telemetry data, and the presence of outliers that may indicate non-human usage (see Table 1), we conducted a further exploratory analysis in which these outliers were removed using the median absolute deviation method (MAD). ${ }^{8}$

provided are based on an alpha of $0.05 / 6$ or 0.00833 . No additional corrections were made for the additional sensitivity analysis models.

${ }^{8}$ The descriptive statistics for the independent variables following this procedure are available in the supplementary materials. 


\section{Results}

The final sample included 8,094 participants and represented individuals who reported residence in seven continents. Of the participants who provided data on their region of residence, a majority indicated that they reside in either Europe (49.10\%) or North America (33.51\%), with the remaining $17.39 \%$ residing in other regions. In terms of age group, for those participants who provided data on their age, the largest group in the sample included those aged between 25 and $29(13.82 \%)$, followed by those aged 65 or greater $(12.15 \%)$. The majority of participants were aged below 40. The distributions for these two demographic covariates are provided in the supplementary materials. Descriptive statistics for the remaining study variables are presented in Table 1. Notably, several variables, particularly URI count and URIs per hour, are extremely right skewed. The maximum values of these variables are illustrative, suggesting that at least one respondent visited more than 60,000 URIs in a week and another visited an average of almost 10,000 URIs per hour of active browser use. It is possible that these extreme values represent automated use (however, all participants completed the survey in-browser, suggesting at least partial organic use), or other browsing patterns that represent a distinct population from most of our participants. After presenting our pre-registered analyses, we address these possibilities in a sensitivity analysis. 
Table 1. Descriptive statistics for key study variables.

\begin{tabular}{lrrrrr}
\hline Variable & $M(S D)$ & Min & Max & Skewness & Kurtosis \\
\hline Active Days & $5.21(1.94)$ & 1.00 & 7.00 & -0.79 & -0.66 \\
Active hours & $8.57(8.95)$ & 0.01 & 156.30 & 3.08 & 23.25 \\
URI count & $1564.83(2144.78)$ & 1.00 & 62529.00 & 6.96 & 114.83 \\
Domain count & $68.40(59.76)$ & 1.00 & 700.00 & 1.96 & 7.31 \\
URI per active hour & $196.76(208.51)$ & 0.05 & 9577.99 & 25.21 & 947.73 \\
Domains per active hour & $11.80(9.20)$ & 0.04 & 144.00 & 3.66 & 25.49 \\
Internet usage desire & $1.87(0.95)$ & 0.00 & 4.00 & 0.18 & -0.20 \\
\hline
\end{tabular}

Note. URI = Uniform Resource Identifier. Active hours = the average active hours per day.

Figure 1 summarizes the responses to the two questions regarding desires to spend more or less time online. Across both versions of the survey, the most likely response was "neither agree nor disagree," and slightly more participants indicated that they wanted to spend less time online rather than more time online. Histograms for the six independent variables are available in the supplementary materials. Table 2 provides a zero-order bivariate correlation matrix for the main study variables. All six of the use metrics are mutually correlated, with the raw metrics indicating larger effect sizes than for the two calculated metrics (domains per active hour and URIs per active hour). While the two "internet usage desire" items were negatively related to each other with a moderate effect size $\left(r_{s}=-0.55, p<0.001\right)$, the magnitude of associations for these variables with all six-usage metrics were small and, in some cases, not statistically significant. 
Distribution of responses to "more/less time online" questions

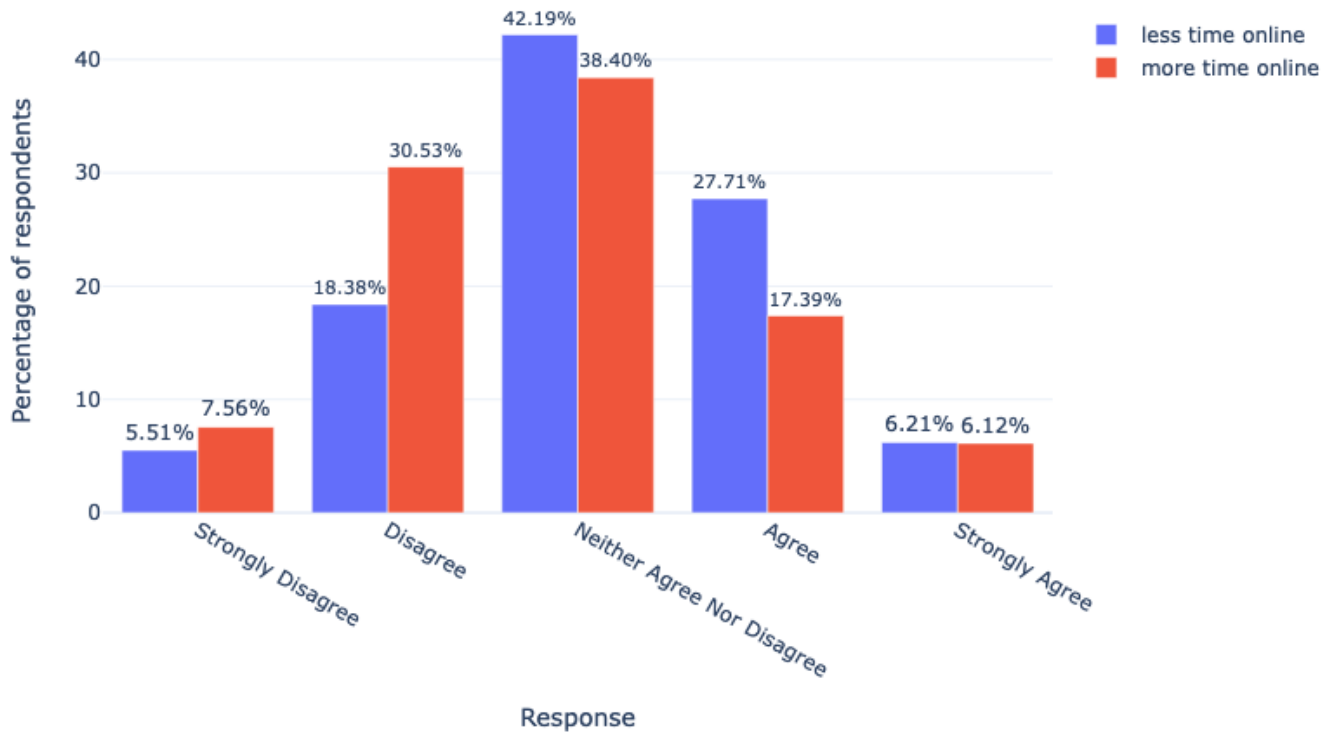

Figure 1. Distribution of responses for the questions about spending more or less time online. 
Table 2. Correlation matrix depicting Spearman correlation coefficients for key study variables.

\begin{tabular}{|c|c|c|c|c|c|c|c|c|}
\hline & 1 & 2 & 3 & 4 & 5 & 6 & 7 & 8 \\
\hline 1. Active days & - & & & & & & & \\
\hline 2. Active hours & $0.68^{* * *}$ & - & & & & & & \\
\hline 3. URI count & $0.61^{* * *}$ & $0.87^{* * *}$ & - & & & & & \\
\hline $\begin{array}{l}\text { 4. Domain } \\
\text { count }\end{array}$ & $0.63^{* * *}$ & $0.80^{* * *}$ & $0.85^{* * *}$ & - & & & & \\
\hline $\begin{array}{l}\text { 5. URIs per } \\
\text { active hour }\end{array}$ & $-0.06^{* * *}$ & $-0.12^{* * *}$ & $0.32^{* * *}$ & $0.16^{* * *}$ & - & & & \\
\hline $\begin{array}{l}\text { 6. Domains per } \\
\text { active hour }\end{array}$ & $-0.30^{* * *}$ & $-0.57^{* * *}$ & $-0.34^{* * *}$ & $-0.04^{* * *}$ & $0.41^{* * *}$ & - & & \\
\hline 7. Age group & $-0.03^{*}$ & $-0.04^{* * *}$ & $-0.10^{* * *}$ & $-0.07^{* * *}$ & $-0.15^{* * *}$ & $-0.03^{* *}$ & - & \\
\hline $\begin{array}{l}\text { 8. More time } \\
\text { online }\end{array}$ & $0.06^{* * *}$ & 0.02 & -0.02 & $-0.05^{* * *}$ & $-0.07^{* * *}$ & $-0.09^{* * *}$ & -0.08 & - \\
\hline $\begin{array}{l}\text { 9. Less time } \\
\text { online }\end{array}$ & $-0.06^{* * *}$ & -0.02 & 0.00 & 0.00 & $0.03^{*}$ & $0.03^{*}$ & -0.02 & $-0.55^{* * *}$ \\
\hline
\end{tabular}

Note. ${ }^{*} p<.05, * * p<.01, * * * p<.001$.

As described in our pre-analysis plan, for each of our independent variables we fitted an ordinal logistic regression model with age group and region included as covariates. Figure 2 depicts the outcomes of these models for each standardized independent variable with a Bonferroni-adjusted confidence interval (indicated in bold red). ${ }^{9}$ In Table 3 we report the specific effect sizes and Bonferroni-adjusted confidence intervals for each variable for the five modeling scenarios. Based on Figure 2 and the values reported in Table 3, it is evident that, for all six variables, effect sizes are very small and, for most variables, the confidence intervals include zero.

\footnotetext{
${ }^{9}$ In a post-hoc exploratory analysis there is a weak signal that the results for domain count may be affected by age, with those in the 25 - 29 age group (the largest) showing different outcomes than those in following groups: 19 - 21, 35 - 39, 40 - 44, 45 - 49. Additionally, those in the 25 - 29 age group differed from those in 35 - 39 age group for URI per hour (see the supplementary materials for the full results of these comparisons). No other age or region interactions for any of the six independent variables were significant.
} 
Table 3. Effect sizes and Bonferroni-adjusted confidence intervals for each variable in each modeling scenario.

$\begin{array}{lrrrr}\text { Variable Pre-registered model } & \text { Including mobile } & \text { Excluding outliers } & \text { Survey with more } & \text { Survey with less } \\ \text { time item }\end{array}$

\begin{tabular}{|c|c|c|c|c|c|}
\hline & $\operatorname{coef}[95 \% \mathrm{CI}]$ & $\operatorname{coef}[95 \% \mathrm{CI}]$ & $\operatorname{coef}[95 \% \mathrm{CI}]$ & $\operatorname{coef}[95 \% \mathrm{CI}]$ & $\operatorname{coef}[95 \% \mathrm{CI}]$ \\
\hline $\begin{array}{l}\text { Days } \\
\text { active }\end{array}$ & $0.1[0.06,0.14]$ & $0.09[0.06,0.13]$ & $0.10[0.06,0.14]$ & $0.06[-0.01,0.13]$ & $0.12[0.05,0.19]$ \\
\hline $\begin{array}{l}\text { Hours } \\
\text { active }\end{array}$ & $0.05[0.01,0.09]$ & $0.06[0.03,0.10]$ & $0.02[-0.02,0.06]$ & $0.02[-0.05,0.09]$ & $0.08[0.01,0.15]$ \\
\hline URI count & $0.01[-0.03,0.05]$ & $0.01[-0.03,0.04]$ & $-0.02[-0.06,0.02]$ & $-0.02[-0.09,0.05]$ & $0.07[0.00,0.14]$ \\
\hline $\begin{array}{l}\text { Domain } \\
\text { count }\end{array}$ & $-0.01[-0.05,0.04]$ & $0.01[-0.02,0.05]$ & $-0.02[-0.06,0.02]$ & $-0.05[-0.12,0.02]$ & $0.08[0.01,0.15]$ \\
\hline $\begin{array}{l}\text { URIs per } \\
\text { active } \\
\text { hour }\end{array}$ & $-0.02[-0.06,0.02]$ & $-0.03[-0.06,0.00]$ & $-0.06[-0.10,-0.01]$ & $-0.01[-0.08,0.06]$ & $0.01[-0.06,0.07]$ \\
\hline $\begin{array}{l}\text { Domains } \\
\text { per active } \\
\text { hour }\end{array}$ & $-0.05[-0.09,-0.01]$ & $-0.04[-0.08,-0.01]$ & $-0.06[-0.10,-0.02]$ & $-0.04[-0.11,0.03]$ & $0.02[-0.05,0.10]$ \\
\hline
\end{tabular}

Coefficient for each standardized IV in internet use desire model with age group and region as control

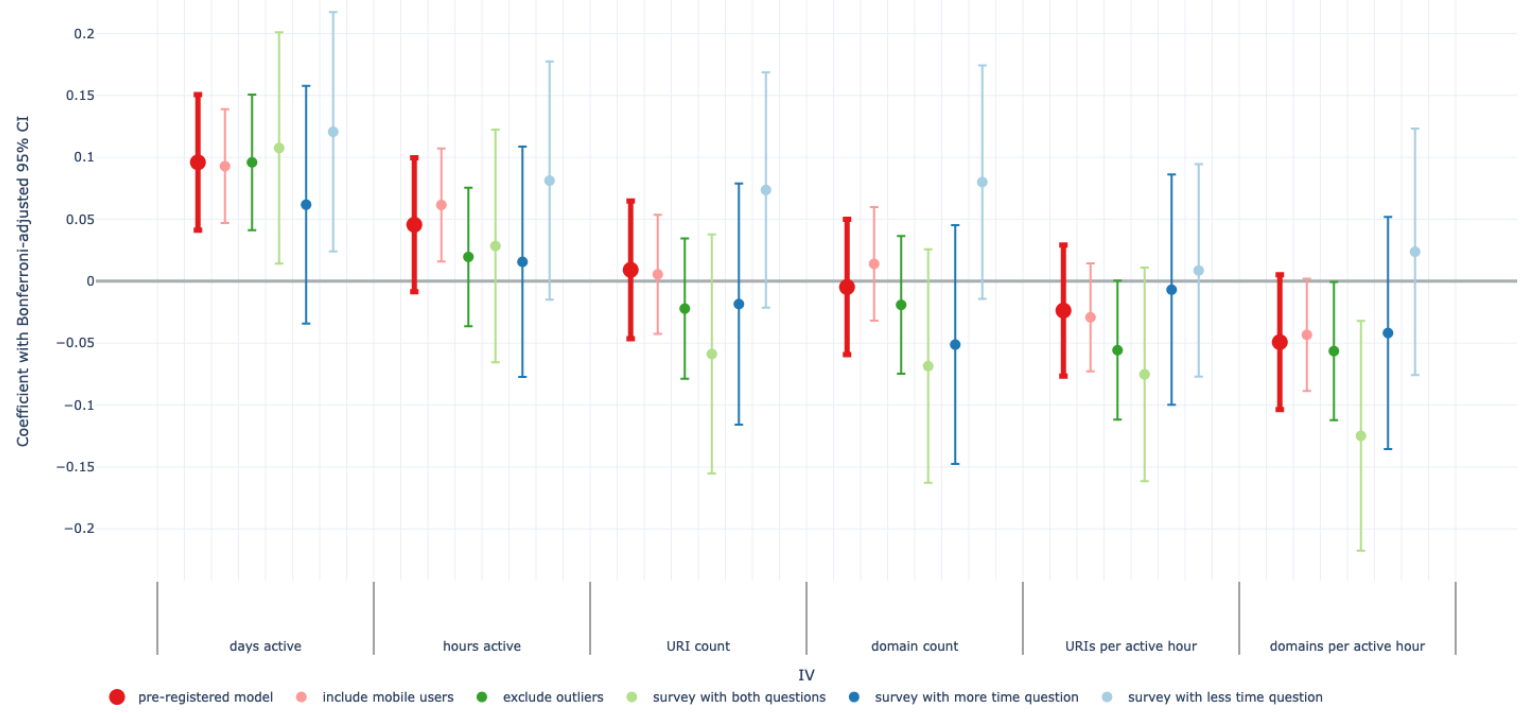

Figure 2. Results of the separate ordinal logistic regression models for each of the six independent variables for the pre-registered and sensitivity analyses. 
Figure 2 also depicts the outcomes for the sensitivity analyses including mobile users, excluding outliers, and analyzing the three different versions of the survey separately. Results for these analyses are comparable to the pre-registered models, with all effect sizes falling in a similar range. Notably, while the confidence intervals were comparable to the pre-registered models, and still tended to include zero, effects were slightly larger and always positive for those who only received the "less time" question (reverse coded and depicted in light blue in Figure 2).

In our final sensitivity analysis, we considered the original response directions separately. As depicted in Figure 3, the pattern of results was comparable with the original pre-specified models, with generally small effect sizes and confidence intervals including zero (except for days active). Notably, effects were in the opposite direction for the "less time" outcome due to the reverse coding in the pre-specified model. While the direction of effects differed between outcomes, the same pattern of statistical significance was found. Except for days active, the confidence intervals for all of the remaining independent variables included zero.

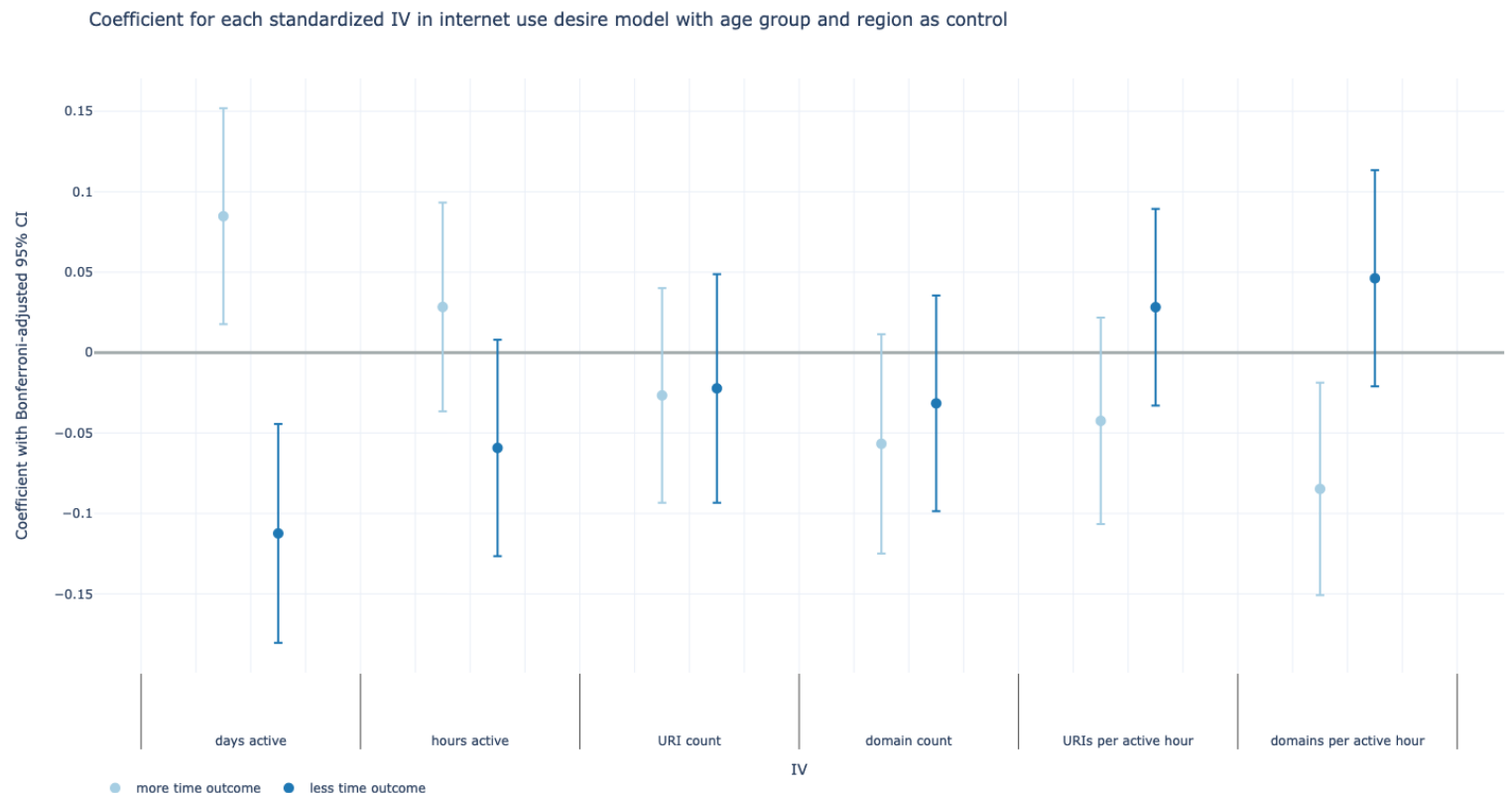

Figure 3. Results of the separate ordinal logistic regression models for the sensitivity analysis 
comparing the "more time online" outcome with the "less time online" outcome.

\section{Discussion}

There is growing recognition that many people feel the need to modulate their use of the internet and other digital technologies in support of their wellbeing (Grady et al., 2022; Hardey \& Atkinson, 2018; Kuntsman \& Miyake, 2019; Lomborg \& Ytre-Arne, 2021; Natale \& Treré, 2020; Nguyen, 2021; Parry et al., 2020). In this pre-registered study, we aimed to advance our understanding of digital well-being in general and, in particular, to study whether browser usage metrics collected by industry partners are associated with desires to regulate time spent online.

To address this aim we used Mozilla Firefox browser telemetry to investigate how six metrics pertaining to time spent on the internet and the diversity and intensity of use predict participants' desires to spend more or less time online. We found that the associations between browser usage and participants' desire to spend more or less time online were very small and, except for active days, we could not reject the null hypothesis of no association. As evidenced by the precision of the confidence intervals around the effect sizes (i.e., tightly bound around the point estimate), our study was well-powered to detect very small effect sizes. Our findings were robust across several different analytical pathways. Browser usage behavior, as indicated by our six metrics, did not appear to be useful for understanding people's desires to spend more or less time online.

Moving beyond high-level browser usage metrics, future research into desires to regulate internet usage should consider the specific content users engage with, the purposes for which they use the internet, and the contexts in which their use occurs, in addition to various individual differences like the user's occupation, experience with the internet, or mental health in general. 
Additionally, as peoples' mindsets towards digital technology usage (e.g., whether they think such usage is "good" or "bad") may relate to how they behave and desires to regulate this behavior (Ernala et al., 2022), there is a need to understand these mindsets and the endogenous (e.g., personal reflections on past experiences online) and exogenous (e.g., media narratives) factors that drive them. Importantly, our measures for desires to spend more or less time online did not attempt to assess any antecedents of these desires. It is likely that various negative perceptions of digital technology use (e.g., distraction, addiction, or other forms of problematic outcomes) or perceptions about one's time allocation (e.g., a lack of discretionary time) account for some of the responses to the survey. In future work investigating the drivers of people's mindsets towards digital technology use, in addition to further validation of our single-item measures, these elements should be considered.

This study addressed concerns about the use of self-report questionnaires to quantify the amount of time spent online in research on digital technology uses and effects and digital wellbeing (Parry et al., 2021). We know that these measures are not accurate, as people cannot reliably estimate the amount of time that they spend using various digital functions (Parry et al., 2021) and, while there remain gaps in our understanding of measurement error (Johannes, Nguyen, et al., 2021), it is likely that the degree of (in)accuracy depends on individual differences that are often fundamental to the effects under investigation (Sewall et al., 2020a; Sewall \& Parry, 2021; Shaw et al., 2020). Additionally, many have expressed their dissatisfaction with the reliance on time-based metrics like the overall amount of time an individual uses digital media (i.e., "screen time"). Although we were limited in the extent to which we could delve into the content of participants' browser usage, our collaboration involving scientific and industry partners enabled us to, firstly, use data recorded directly from browser 
usage sessions and, secondly, extend the range of ways to measure how people use their browsers. This enabled us to investigate effects associated with actual browser usage intensity and diversity alongside other time-based metrics. Notwithstanding these contributions, while we used objective measures for browser usage, our dependent variables — desires to spend more or less time online - were assessed with single-item measures. This is a limitation and future work is needed to investigate the validity of these measures.

In addition to addressing the primary research question, the findings provide an indication of the proportion of individuals who feel the need to self-regulate their internet use. Using a relatively large, albeit non-representative, sample of Firefox users, the results show that most participants did not indicate strong desires to spend either more or less time online. Only a small proportion of participants indicated strong agreement with either the desire to spend less time online or the desire to spend more time online. This finding suggests that most people are generally satisfied with, or indifferent towards, the amount of time that they spend online and, therefore, that they do not feel a need to regulate this behavior in pursuit of some other goal or desired state. Alternatively, the findings may also be reflective of successful self-regulationpeople are satisfied with the current state of their behavior and may already be using various preventive or interventive strategies to achieve this state. Either way, given that our sample was relatively young and included users of only a single web browser, further research is needed to determine the extent to which these findings generalize to older populations and users of other web browsers.

Irrespective of the mechanism, the findings suggest that, just as digital technology effects are likely to be highly heterogeneous, with only a small proportion of users experiencing either positive or negative outcomes (Beyens et al., 2020; Valkenburg, Beyens, et al., 2021), desires to 
regulate digital technology usage follow a similar pattern, with most users satisfied or indifferent to the amount of time they spend online, and only a small proportion wanting change. However, to extend our correlational, between-person findings and further understand how aspects of browsing behavior relate to desires to regulate digital technology use, there is a need to investigate intra-individual consistency in browsing behaviors (e.g., via intensive longitudinal studies or repertoire approaches; Horvát \& Hargittai, 2021; Parry \& Sewall, 2021; Valkenburg, Pouwels, et al., 2021) and determine how the variability in desires to regulate behavior relates, firstly, to the consistency of browsing behavior and, secondly, to particular "types" of browsing. Such work will not only further our understanding of digital wellbeing but, more generally, enable us to learn about the stability of everyday behaviors (e.g., O’Connor \& Rosenblood, 1996) and the frames with which individuals view these behaviors (Hofmann et al., 2012).

\section{Challenges associated with the use of telemetry}

The study also highlights several challenges inherent to using telemetry or trace data to draw inferences with user desires, characteristics, outcomes, or perspectives. New ways of measurement provide new opportunities for understanding human behavior, but they also present new challenges and require careful calibration for these opportunities to be realized.

The first challenge concerns the distinction between "readymade" data and “custommade" data. Salganik (2019) draws on the "readymades," a series of artworks by the French artist Marcel Duchamp, to distinguish between data that were produced specifically for research purposes in a pre-planned manner (i.e., "custommade" data), and data that were generated for one purpose which are then repurposed to address a research objective for which

they were not originally generated (i.e., "readymade" data). This distinction highlights the fact 
that repurposed, "readymade" data may not always fit the characteristics needed for a study, and that, while such data can address research questions in ways "custommade" data cannot, one needs to be aware of, and account for, the data generating processes inherent to the source.

Behavioral data on digital technology use, whether acquired through industry-academia collaborations (as in this study), custom trackers, or third-party APIs or trackers, will invariably draw on telemetry systems not originally produced for research purposes. Not only does this mean that such data sources may be biased by various individual and technological factors (Jürgens et al., 2019; Parry et al., 2021; Scharkow, 2016) but it also implies that there may be a mismatch between the existing data and the behavior that researchers want to measure based on a theoretical model (i.e., usage content, diversity, or intensity for specific applications or services). In a related manner, analyses may be driven by the data that are available, rather than the data that are truly needed to address a research question. For instance, in this study we were limited in that we could only access and study desktop browser usage. It is likely that a substantial proportion of browser usage takes place on a smartphone or via dedicated applications. Such behavior was out of reach to us. Assessment of digital technology usage across hardware devices or even across various platforms or modalities remains a key challenge in this domain.

A second challenge associated with the use of telemetry or trace data concerns the calibration and validation of the measures. In this context, calibration refers to the extent to which a measure captures the intended actions (i.e., whether a measure records human actions as well as other automated or background processes). While it is well established that usage logs do not correlate with estimates of usage (Parry et al., 2021), little research has focused on the validation of telemetric mechanisms for recording digital technology usage (c.f., Elhai et al., 2018; Geyer et al., 2021). In the context of smartphone usage logs, concerns have been raised 
that such systems can mis-record background functions as active usage, or that some forms of active usage (e.g., interactions via 'lock-screens') may not be logged at all (Jürgens et al., 2019). It is reasonable to assume that the accuracy of telemetry-based metrics for internet usage may also suffer from various biases and technical challenges. As more researchers leverage telemetry to measure behavior with digital technologies, the validation of the tools used to produce these measures becomes a key concern.

Telemetry and trace data can be of variable quality, as the data are typically captured with minimal researcher involvement. It can be difficult to differentiate data that are "natural" (i.e., data that represent typical use patterns by a user on the platform) from data that are "unnatural" (i.e., data that represent a use pattern that is impossible or highly improbable). In this study, several browser usage metrics had substantially skewed distributions with several participants' usage far exceeding the averages. This presented a challenge for understanding whether these values represented measurement error, natural human usage, or unnatural usage by automated agents. Future studies leveraging telemetry systems to measure digital technology use will likely face similar challenges. In addition to the need for research into the accuracy and calibration of these tracking systems, variability in data quality and poorly understood usage patterns also signal the need for pre-registration to guard against $p$-hacking in search of statistically significant results (Dienlin et al., 2020). In-depth pre-registration and sensitivitychecking procedures based on a more complete understanding of what actual human digital technology usage looks like can provide guide rails to help researchers process behavioral data without succumbing to the garden of forking paths. It will also be increasingly important for 
researchers to calibrate their measures to truly understand whether they are measuring the conceptualisations they are aimed at, and account for the inevitable imperfections.

\section{Conclusion}

To advance our understanding of digital well-being and study the usage factors that may be associated with desires to regulate time spent online, this study drew on browser telemetry collected in collaboration with the Mozilla Corporation, the developers of the Firefox browser. In addition to addressing our primary research question and showing that actual browser usage metrics, at a high-level, do not relate to desires to self-regulate the amount of time spent online, the study also highlights a number of considerations and concerns that need to be addressed in future industry-academia collaborations that draw on trace data or usage telemetry. Despite these challenges, we are optimistic about the potential inherent in telemetry and trace data for enhancing our understanding of the role of digital technologies in human behavior and wellbeing (Lazer et al., 2021). Central to realizing this potential will be increased collaboration between academia and the technology companies that develop and maintain the platforms and services through which large parts of our lives are mediated. 


\section{References}

Allcott, H., Gentzkow, M., \& Song, L. (2021). Digital Addiction. http://www.nber.org/dataappendix/w28936

Araujo, T., Wonneberger, A., Neijens, P., \& de Vreese, C. (2017). How Much Time Do You Spend Online? Understanding and Improving the Accuracy of Self-Reported Measures of Internet Use. Communication Methods and Measures, 11(3), 173-190. https://doi.org/10.1080/19312458.2017.1317337

Beyens, I., Pouwels, J. L., van Driel, I. I., Keijsers, L., \& Valkenburg, P. M. (2020). The effect of social media on well-being differs from adolescent to adolescent. Scientific Reports. https://doi.org/10.1038/s41598-020-67727-7

Büchi, M. (2020). Digital Well-Being Theory and Research [Preprint]. Open Science Framework. https://doi.org/10.31219/osf.io/k3e2j

Chib, A., Ang, M. W., Ibasco, G. C., \& Nguyen, H. (2021). Mobile Media (Non-)Use as Expression of Agency. Mass Communication and Society, 24(6), 818-842. https://doi.org/10.1080/15205436.2021.1970187

Dickson, K., Richardson, M., Kwan, I., Macdowall, W., Burchett, H., Stansfield, C., Brunton, G., Sutcliffe, K., \& Thomas, J. (2018). Screen-based activities and children and young people's mental health and psychosocial wellbeing: A systematic map of reviews. Department of Health Reviews Facility. http://eppi.ioe.ac.uk/cms/Default.aspx?tabid=3748

Dienlin, T., Johannes, N., Bowman, N. D., Masur, P. K., Engesser, S., Kümpel, A. S., Lukito, J., Bier, L. M., Zhang, R., Johnson, B. K., Huskey, R., Schneider, F. M., Breuer, J., Parry, D. A., Vermeulen, I., Fisher, J. T., Banks, J., Weber, R., Ellis, D. A., ... de Vreese, C. 
(2020). An Agenda for Open Science in Communication. Journal of Communication, 26. https://doi.org/10.1093/joc/jqz052

Duckworth, A. L., Gendler, T. S., \& Gross, J. J. (2016). Situational Strategies for Self-Control. Perspectives on Psychological Science, 11(1), 35-55. https://doi.org/10.1177/1745691615623247

Duckworth, A. L., Taxer, J. L., Eskreis-Winkler, L., Galla, B. M., \& Gross, J. J. (2019). SelfControl and Academic Achievement. Annual Review of Psychology, 70(1), 373-399. https://doi.org/10.1146/annurev-psych-010418-103230

Elhai, J. D., Tiamiyu, M. F., Weeks, J. W., Levine, J. C., Picard, K. J., \& Hall, B. J. (2018). Depression and emotion regulation predict objective smartphone use measured over one week. Personality and Individual Differences, 133, 21-28. https://doi.org/10.1016/j.paid.2017.04.051

Ellis, D. A. (2019). Are smartphones really that bad? Improving the psychological measurement of technology-related behaviors. Computers in Human Behavior, 97(March), 60-66. https://doi.org/10.1016/j.chb.2019.03.006

Ernala, S. K., Burke, M., Leavitt, A., \& Ellison, N. B. (2022). Mindsets Matter: How Beliefs About Facebook Moderate the Association Between Time Spent and Well-Being. CHI Conference on Human Factors in Computing Systems, 1-13. https://doi.org/10.1145/3491102.3517569

Festic, N., Büchi, M., \& Latzer, M. (2021). How Long and What For? Tracking a Nationally Representative Sample to Quantify Internet Use. Journal of Quantitative Description: Digital Media, 1. https://doi.org/10.51685/jqd.2021.018

Freelon, D. (2014). On the Interpretation of Digital Trace Data in Communication and Social 
Computing Research. Journal of Broadcasting \& Electronic Media, 58(1), 59-75. https://doi.org/10.1080/08838151.2013.875018

Fu, S., Li, H., Liu, Y., Pirkkalainen, H., \& Salo, M. (2020). Social media overload, exhaustion, and use discontinuance: Examining the effects of information overload, system feature overload, and social overload. Information Processing \& Management, 57(6), 102307. https://doi.org/10.1016/j.ipm.2020.102307

Gazzaley, A. (2018). The Cognition Crisis [Blog]. Elemental. https://elemental.medium.com/thecognition-crisis-a1482e $889 \mathrm{fcb}$

Geyer, K., Ellis, D. A., Shaw, H., \& Davidson, B. I. (2021). Open-source smartphone app and tools for measuring, quantifying, and visualizing technology use. Behavior Research Methods. https://doi.org/10.3758/s13428-021-01585-7

Grady, S. M., Eden, A., Johnson, B. K., \& Reinecke, L. (2022). Media use and avoidance experiences during social distancing. Technology, Mind, and Behavior, 3(1). https://doi.org/10.1037/tmb0000041

Granic, I., Morita, H., \& Scholten, H. (2020). Beyond Screen Time: Identity Development in the Digital Age. Psychological Inquiry, 31(3), 195-223. https://doi.org/10.1080/1047840X.2020.1820214

Griffioen, N., van Rooij, M., Lichtwarck-Aschoff, A., \& Granic, I. (2020). Toward improved methods in social media research. Technology, Mind, and Behavior, 1(1). https://doi.org/10.1037/tmb0000005

Hardey, M., \& Atkinson, R. (2018). Disconnected: Non-Users of Information Communication Technologies. Sociological Research Online, 23(3), 553-571. https://doi.org/10.1177/1360780418764736 
Harris, T. (2016). How Technology is Hijacking Your Mind-From a Magician and Google Design Ethicist. Thrive Global. https://medium.com/thrive-global/how-technologyhijacks-peoples-minds-from-a-magician-and-google-s-design-ethicist-56d62ef5edf3

Hesselberth, P. (2018). Discourses on disconnectivity and the right to disconnect. New Media \& Society, 20(5), 1994-2010. https://doi.org/10.1177/1461444817711449

Hofmann, W., Vohs, K. D., \& Baumeister, R. F. (2012). What People Desire, Feel Conflicted About, and Try to Resist in Everyday Life. Psychological Science, 23(6), 582-588. https://doi.org/10.1177/0956797612437426

Horvát, E.-Á., \& Hargittai, E. (2021). Birds of a Feather Flock Together Online: Digital Inequality in Social Media Repertoires. Social Media + Society, 7(4), 205630512110528. https://doi.org/10.1177/20563051211052897

Inzlicht, M., Werner, K. M., Briskin, J. L., \& Roberts, B. W. (2021). Integrating Models of SelfRegulation. Annual Review of Psychology, 72(1), 319-345. https://doi.org/10.1146/annurev-psych-061020-105721

Johannes, N., \& Dienlin, T. (2020). The impact of digital technology use on adolescent wellbeing. Dialogues in Clinical Neuroscience, 22(2), 135-142. https://doi.org/10.31887/DCNS.2020.22.2/tdienlin

Johannes, N., Nguyen, T., Weinstein, N., \& Przybylski, A. K. (2021). Objective, subjective, and accurate reporting of social media use: No evidence that daily social media use correlates with personality traits, motivational states, or well-being. Technology, Mind, and Behavior, 2(2). https://doi.org/10.1037/tmb0000035

Johannes, N., Vuorre, M., \& Przybylski, A. K. (2021). Video game play is positively correlated with well-being. Royal Society Open Science, 8(2), rsos.202049, 202049. 
https://doi.org/10.1098/rsos.202049

Jorge, A. (2019). Social Media, Interrupted: Users Recounting Temporary Disconnection on Instagram. Social Media + Society, 5(4), 205630511988169. https://doi.org/10.1177/2056305119881691

Jürgens, P., Stark, B., \& Magin, M. (2019). Two Half-Truths Make a Whole? On Bias in SelfReports and Tracking Data. Social Science Computer Review. https://doi.org/10.1177/0894439319831643

Kaye, L. K., Orben, A., Ellis, D. A., Hunter, S. C., \& Houghton, S. (2020). The conceptual and methodological mayhem of "screen time." International Journal of Environmental Research and Public Health, 17, 3661. https://doi.org/10.3390/ijerph17103661

Kuntsman, A., \& Miyake, E. (2019). The paradox and continuum of digital disengagement: Denaturalising digital sociality and technological connectivity. Media, Culture \& Society, 41(6), 901-913. https://doi.org/10.1177/0163443719853732

Lazer, D., Hargittai, E., Freelon, D., Gonzalez-Bailon, S., Munger, K., Ognyanova, K., \& Radford, J. (2021). Meaningful measures of human society in the twenty-first century. Nature, 595(7866), 189-196. https://doi.org/10.1038/s41586-021-03660-7

Lin, J., Lin, S., Turel, O., \& Xu, F. (2020). The buffering effect of flow experience on the relationship between overload and social media users' discontinuance intentions. Telematics and Informatics, 49, 101374. https://doi.org/10.1016/j.tele.2020.101374

Lomborg, S., \& Ytre-Arne, B. (2021). Advancing digital disconnection research: Introduction to the special issue. Convergence: The International Journal of Research into New Media Technologies, 135485652110575. https://doi.org/10.1177/13548565211057518

Marder, B., Houghton, D., Joinson, A., Shankar, A., \& Bull, E. (2016). Understanding the 
Psychological Process of Avoidance-Based Self-Regulation on Facebook. Cyberpsychology, Behavior, and Social Networking, 19(5), 321-327. https://doi.org/10.1089/cyber.2015.0564

Meier, A., \& Reinecke, L. (2020). Computer-mediated communication, social media, and mental health: A conceptual and empirical meta-review. Communication Research, 1-28. https://doi.org/10.31234/osf.io/573ph

Mozilla. (2017). Mozilla Source Tree Docs: Telemetry. https://firefox-sourcedocs.mozilla.org/toolkit/components/telemetry/ telemetry/index.html Mozilla. (2021a). Firefox Probe Dictionary_Find Probes [Documentation]. https://probes.telemetry.mozilla.org/ $?$ search=active $+\& v i e w=$ detail $\&$ probeId $=$ scalar $\% 2 \mathrm{Fb}$ rowser.engagement.active_ticks

Mozilla. (2021b). Metrics-Mozilla Data Documentation: Daily Active Users (DAU) [Documentation]. Mozilla Data Documentation. https://docs.telemetry.mozilla.org/metrics/metrics.html\#daily-active-users-dau

Natale, S., \& Treré, E. (2020). Vinyl won't save us: Reframing disconnection as engagement. Media, Culture \& Society, 42(4), 626-633. https://doi.org/10.1177/0163443720914027

Nguyen, M. H. (2021). Managing Social Media Use in an “Always-On” Society: Exploring Digital Wellbeing Strategies That People Use to Disconnect. Mass Communication and Society, 24(6), 795-817. https://doi.org/10.1080/15205436.2021.1979045

Nowok B, Raab GM, Dibben C. 2016. Synthpop:bespoke creation of synthetic data in R. Journal of Statistical Software 74:1-26. DOI: https://doi.org/10.18637/jss.v074.i11.

O’Connor, S. C., \& Rosenblood, L. K. (1996). Affiliation motivation in everyday experience: A theoretical comparison. Journal of Personality and Social Psychology, 70(3), 513-522. 
https://doi.org/10.1037/0022-3514.70.3.513

Orben, A. (2020a). Teenagers, screens and social media: A narrative review of reviews and key studies. Social Psychiatry and Psychiatric Epidemiology, 55(4), 407-414. https://doi.org/10.1007/s00127-019-01825-4

Orben, A. (2020b). The Sisyphean Cycle of Technology Panics. Perspectives on Psychological Science, 1745691620919372. https://doi.org/10.1177/1745691620919372

Orben, A., \& Przybylski, A. K. (2020). Reply to: Underestimating digital media harm. Nature Human Behaviour, 4, 349-351. https://doi.org/10.1038/s41562-020-0840-y

Orben, A., Weinstein, N., \& Przybylski, A. K. (2020). Only Holistic and Iterative Change Will Fix Digital Technology Research. Psychological Inquiry, 31(3), 235-241. https://doi.org/10.1080/1047840X.2020.1820221

Orhan, M. A., Castellano, S., Khelladi, I., Marinelli, L., \& Monge, F. (2021). Technology distraction at work. Impacts on self-regulation and work engagement. Journal of Business Research, 126, 341-349. https://doi.org/10.1016/j.jbusres.2020.12.048

Parks, M. (2021, May 18). Facebook Calls Links to Depression Inconclusive. These Researchers Disagree. Npr. https://www.npr.org/2021/05/18/990234501/

Parry, D., Davidson, B. I., Sewall, C. J. R., Fisher, J. T., Mieczkowski, H., \& Quintana, D. S. (2021). A systematic review and meta-analysis of discrepancies between logged and selfreported digital media use. Nature Human Behaviour, 1-13. https://doi.org/10.1038/s41562-021-01117-5

Parry, D., Fisher, J. T., Mieczkowski, H., Sewall, C. J. R., \& Davidson, B. I. (2022). Social Media and Well-being: A Methodological Perspective. Current Opinion in Psychology, 45. https://doi.org/10.1016/j.copsyc.2021.11.005 
Parry, D., le Roux, D., Morton, J., Pons, R., Pretorius, R., \& Schoeman, A. (2020). Digital Wellbeing Applications: Adoption, Use and Perceived Effects [Preprint]. SocArXiv. https://doi.org/10.31235/osf.io/6e9ap

Parry, D., \& Sewall, C. (2021). How consistent are smartphone application preferences? A longitudinal study of mobile application repertoires using behavioral data. SocArXiv. https://doi.org/10.31235/osf.io/jzxun

Pelet, J.-É., Ettis, S., \& Cowart, K. (2017). Optimal experience of flow enhanced by telepresence: Evidence from social media use. Information \& Management, 54(1), 115128. https://doi.org/10.1016/j.im.2016.05.001

Portwood-Stacer, L. (2013). Media refusal and conspicuous non-consumption: The performative and political dimensions of Facebook abstention. New Media \& Society, 15(7), 10411057. https://doi.org/10.1177/1461444812465139

Przybylski, A. K., Johannes, N., Vuorre, M., Dodel, M., Gottlieb, C., Kaye, L. K., Davidson, B. I., Ellis, D. A., Parry, D. A., Odgers, C. L., Sewall, C. J. R., Etchells, P. J., Abbas, R., Boniel-Nissim, M., Law, W., Erhel, S., Howard, A. L., Modecki, K., \& Orben, A. (2021). An Open Letter to Mr. Mark Zuckerberg: A Global Call to Act Now on Child and Adolescent Mental Health Science. https://www.oii.ox.ac.uk/an-open-letter-to-markzuckerberg

Quintana, D. S. (2020). A synthetic dataset primer for the biobehavioural sciences to promote reproducibility and hypothesis generation. ELife, 9, e53275. https://doi.org/10.7554/eLife.53275

Reinecke, L., Gilbert, A., \& Eden, A. (2021). Self-Regulation as a Key Boundary Condition in the Relationship Between Social Media Use and Well-Being. Current Opinion in 
Psychology, S2352250X21002487. https://doi.org/10.1016/j.copsyc.2021.12.008

Reiter JP (2005). Using CART to Generate Partially Synthetic, Public Use Microdata. Journal of Official Statistics, 21(3), 441-462.

Rogers, K. (2021, July 7). Phone and social media “addiction”: 12 ways to address it. CNN.Com. https://www.cnn.com/2021/07/07/health/phone-addiction-tips-definitionwellness/index.html

Salganik, M. (2019). Bit by Bit: Social Research in the Digital Age. Princeton University Press.

Scharkow, M. (2016). The Accuracy of Self-Reported Internet Use-A Validation Study Using Client Log Data. Communication Methods and Measures, 10(1), 13-27. https://doi.org/10.1080/19312458.2015.1118446

Schwarz, N., \& Oyserman, D. (2001). Asking questions about behavior. American Journal of Evaluation, 22(2), 127-160. https://doi.org/10.1177/109821400102200202

Sewall, C. J. R., Bear, T. M., Merranko, J., \& Rosen, D. (2020a). How psychosocial well-being and usage amount predict inaccuracies in retrospective estimates of digital technology use. Mobile Media and Communication, 8(3), 379-399. https://doi.org/10.1177/2050157920902830

Sewall, C. J. R., Bear, T. M., Merranko, J., \& Rosen, D. (2020b). How psychosocial well-being and usage amount predict inaccuracies in retrospective estimates of digital technology use. Mobile Media \& Communication, 205015792090283. https://doi.org/10.1177/2050157920902830

Sewall, C. J. R., \& Parry, D. (2021). The Role of Depression in the Discrepancy Between Estimated and Actual Smartphone Use: A Cubic Response Surface Analysis. Technology, Mind, and Behavior, 9. 
Shaw, H., Ellis, D. A., Geyer, K., Davidson, B., Ziegler, F., \& Smith, A. (2020). Quantifying smartphone "use": Choice of measurement impacts relationships between "usage" and health. Technology, Mind, and Behavior. https://doi.org/10.1037/tmb0000022

Tourangeau, R. (1984). Cognitive science in survey methods. In T. Jabine, M. Straf, J. Tanur, \& R. Tourangeau (Eds.), Cognitive aspects of survey design: Building a bridge between disciplines (pp. 73-100). The National Academies Press. https://doi.org/10.17226/930

Twenge, J. M. (2018). Have Smartphones Destroyed a Generation? The Atlantic.

Twenge, J. M., Haidt, J., Joiner, T. E., \& Campbell, W. K. (2020). Underestimating digital media harm. Nature Human Behaviour, 4(April), 346-349. https://doi.org/10.1038/s41562-020$0839-4$

Valkenburg, P. M., Beyens, I., Pouwels, J. L., \& Driel, I. I. van. (2021). Social media use and adolescents' self-esteem: Heading for a person-specific media effects paradigm. Journal of Communication, 2020. https://doi.org/10.1093/joc/jqaa039

Valkenburg, P. M., Meier, A., \& Beyens, I. (2022). Social media use and its impact on adolescent mental health: An umbrella review of the evidence. Current Opinion in Psychology, 44, 58-68. https://doi.org/10.1016/j.copsyc.2021.08.017

Valkenburg, P. M., Pouwels, J. L., Beyens, I., van Driel, I. I., \& Keijsers, L. (2021). Adolescents' social media experiences and their self-esteem: A person-specific susceptibility perspective. Technology, Mind, and Behavior, 2(2). https://doi.org/10.1037/tmb0000037

Vanden Abeele, M. M. P. (2020). Digital Wellbeing as a Dynamic Construct. Communication Theory, qtaa024. https://doi.org/10.1093/ct/qtaa024

Ytre-Arne, B., Syvertsen, T., Moe, H., \& Karlsen, F. (2020). Temporal ambivalences in 
smartphone use: Conflicting flows, conflicting responsibilities. New Media \& Society, 22(9), 1715-1732. 